\title{
DERIVATIVE-TYPE ASCENT FORMULAS FOR KERNELS OF SOME HALF-SPACE DIRICHLET PROBLEMS
}

\author{
L. R. BRAGG ${ }^{1}$ \\ (Received 6 September 1996; revised 7 February 2000)

\begin{abstract}
Derivative-type ascent formulas are deduced for the kernels of certain half-space Dirichlet problems. These have the character of differentiation formulas for the Bessel functions but involve modifying variables after completing the differentiations. The Laplace equation and the equation of generalized axially-symmetric potential theory (GASPT) are considered in these. The methods employed also permit treating abstract versions of Dirichlet problems.
\end{abstract}

\section{Introduction}

In studies of the wave and other hyperbolic initial value problems, Hadamard [9] introduced the method of descent. To solve a wave problem in an even number of space dimensions, he first solved an associated problem with one additional space dimension and then projected out the extra variable by integration. This permitted him to develop kernel functions for these even-space dimensional wave problems. He described this approach by "he who can do more can do less". This approach has been extended by others but the main difficulty is "doing the more" before tackling "the less". An alternative to this is the ascent approach whereby one seeks to solve problems involving several space variables from solutions of similar problems having fewer such variables. In [6], Bureau added space variables into the solution of a lower-order wave problem and then carried out smoothing operations on this to obtain the solution of a higher-dimensional wave problem. The operational calculus together with developments in semigroup theory have led to developing integral ascent formulas for solving regular and singular initial value problems, Dirichlet problems and abstract differential equation problems $([2,4]$ and $[10])$. In these cases, the introduction of additional variables (or operators) was effected by means of real- and complex-type convolutions on solutions of associated single variable (or operator) problems. Ascent

${ }^{\text {I}}$ Department of Mathematics and Statistics, Oakland University, Rochester, MI 48309-4485, USA.

(C) Australian Mathematical Society 2000, Serial-fee code 0334-2700/00 
methods for other boundary problems have been discussed by Gilbert [8] and Norwood [12]. In this paper, we treat ascent for a variety of Dirichlet problems from a different view point by constructing repeated derivative-type ascent formulas for their kernels. This permits expressing kernels for higher-dimensional problems in terms of a few starting functions. We now introduce notation and background for this for classical half-space Dirichlet problems.

Let $x=\left(x_{1}, x_{2}, \ldots, x_{n}\right), \xi=\left(\xi_{1}, \xi_{2}, \ldots, \xi_{n}\right)$ and $\|x\|_{n}^{2}=\sum_{j=1}^{n} x_{j}^{2}$. Take $c_{1}$, $c_{2}, \ldots, c_{n}$ and $a$ to be real parameters with $a \geq 0$ and let $D_{j}=\partial / \partial x_{j}, j=1, \ldots, n$. We are concerned with the structure formulas for kernel functions $k_{n}(x, y)$ (or, alternatively, $\left.\tilde{k}_{n}(x, y)\right)$ associated with certain half-space Dirichlet problems. These include

$$
\begin{aligned}
& v_{y y}(x, y)+\Delta_{n} v(x, y)+\sum_{j=1}^{n} 2 c_{j} D_{j} v(x, y)-a^{2} v(x, y)=0, \quad y>0, \\
& v(x, 0+)=\phi(x),
\end{aligned}
$$

where $\Delta_{n}$ denotes the Laplacian operator $\sum_{j=1}^{n} D_{j}^{2}$. With this $k_{n}$, we have

$$
v(x, y)=\int_{E_{n}} k_{n}(x-\xi, y) \phi(\xi) d \xi,
$$

where $E_{n}$ denotes Euclidean $n$-space and $d \xi$ denotes its element of volume. Using transmutations connecting solutions of initial value heat problems to these types of Dirichlet problems $([1,5])$ and the properties of the Laplace transform, ascent formulas for the functions $k_{n}(x, y)$ are obtained that involve repeated derivatives of expressions involving the modified Bessel functions $K_{v}$. Their precise forms depend upon the parity of $n$. For example, the choices $n=2 m$ and $c_{j}=0, j=1,2, \ldots, n$, lead to the formula

$$
k_{2 m}(x, y)=\left.y \cdot\left(-\pi^{-1} D_{r}\right)^{m}\left\{r^{-1 / 2} e^{-a r^{1 / 2}}\right\}\right|_{r=y^{2}+\|x\|_{2 m}^{2}},
$$

where $D_{r}=\partial / \partial r$. The right member of this has an "ascent" character in the sense that each differentiation increases by two the number of terms $x_{j}^{2}$ that appear in the expression for $r$. The dependence of $r$ upon the number of differentiations makes evident the difference between the derivative form in (1.3) and the Rodrigue-type formulas for the Hermite and other special polynomials (see [11]). When $a=0$, a simple calculation in (1.3) yields the familiar Dirichlet kernel function

$$
k_{2 m}(x, y)=\frac{(2 m) !}{(4 \pi)^{m} m !} y\left[y^{2}+\|x\|_{2 m}^{2}\right]^{-(m+1 / 2)} .
$$

For deducing these ascent formulas, we recall, in Section 2, transmutations that relate solutions of generalized heat problems to solutions of the above Dirichlet 
problems. The first is employed in Sections 3 and 4 to develop the ascent forms for the kernel functions for the problem (1.1) first when $n=2 m$ and then when $n=2 m+1$. In this second case, the kernel is expressible in terms of the modified Bessel function $K_{1}$. For certain choices of the constants $c_{j}$, it is necessary to restrict the support or the growth of the data function $\phi(x)$ to ensure that the integral in (1.2) exists. At the end of Section 4, we note a connection between some of these formulas and "descent". In Section 5 we note the corresponding derivative ascent formulas for kernels of Dirichlet type problems that involve the equation of generalized axially-symmetric potential theory (GASPT). Finally, in Section 6, we treat an abstract version of the Dirichlet problem and then apply these ascent formulas to solve a specific example.

\section{Basic transmutations}

With $x$ as above, take $D=\left(D_{1}, D_{2}, \ldots, D_{n}\right)$ and let $P(x, D)$ denote a strongly elliptic operator. We recall from [5] that if the function $u(x, t)$ is a bounded continuous solution of the "generalized" heat problem

$$
\begin{aligned}
& u_{t}(x, t)=P(x, D) u(x, t), \quad t>0 \\
& u(x, 0+)=\phi(x),
\end{aligned}
$$

with $\phi(x)$ continuous, then the function

$$
v(x, y)=\frac{y}{\sqrt{\pi}} \int_{0}^{\infty} e^{-y^{2} \sigma} \sigma^{-1 / 2} u(x, 1 /(4 \sigma)) d \sigma
$$

is a bounded solution of the Dirichlet problem

$$
\begin{aligned}
& v_{y y}(x, y)+P(x, D) v(x, y)=0, \quad y>0 ; \\
& v(x, 0+)=\phi(x) .
\end{aligned}
$$

Similarly, if $\mu<1$, it follows from [1] that the function

$$
U(x, y)=\frac{y^{1-\mu}}{\Gamma((1-\mu) / 2)} \int_{0}^{\infty} e^{-y^{2} \sigma} \sigma^{-(\mu+1) / 2} u(x, 1 /(4 \sigma)) d \sigma
$$

is a solution of the singular Dirichlet problem

$$
\begin{aligned}
& U_{y y}(x, y)+\frac{\mu}{y} U_{y}(x, y)+P(x, D) U(x, y)=0, \quad y>0 ; \\
& U(x, 0)=\phi(x) .
\end{aligned}
$$

Using the theory of distributions, it has been established that these transmutations transform fundamental solutions into fundamental solutions [7]. In particular, the 
integral operators in (2.2) and (2.4) transform the kernel function of (2.1) into the kernel functions for the respective problems (2.3) and (2.5). We will make use of this fact in the sections to follow.

Finally, if $\psi\left(x_{1}, x_{2}, \ldots, x_{n}\right)$ has continuous first partial derivatives with respect to each of the variables $x_{j}, j=1,2, \ldots, n$ and if $b_{1}, b_{2}, \ldots, b_{n}$ are real parameters independent of the $x_{j}$, then we have the exponential translation relation

$$
e^{\sum_{j=1}^{n} b_{j} D_{j}} \psi\left(x_{1}, x_{2}, \ldots, x_{n}\right)=\psi\left(x_{1}+b_{1}, x_{2}+b_{2}, \ldots, x_{n}+b_{n}\right)
$$

\section{Kernel for (1.1) when $n=2 m$}

Consider first the Dirichlet problem (1.1) with $n$ even. We establish the formula (1.3) corresponding to $c_{j}=0$ for all $j$. A "heat type" problem related to this is given by

$$
\begin{aligned}
& u_{t}(x, t)=\Delta_{2 m} u(x, t)-a^{2} u(x, t), \quad t>0 \\
& u(x, 0)=\phi(x)
\end{aligned}
$$

This has the symbolic and analytic solution

$$
\begin{aligned}
u(x, t) & =e^{t\left(\Delta_{2 m}-a^{2}\right)} \phi(x)=e^{-a^{2} t} e^{t \Delta_{2 m}} \phi(x) \\
& =\frac{e^{-a^{2} t}}{(4 \pi t)^{m}} \int_{E_{2 m}} e^{-\|x-\xi\|_{2 m}^{2} /(4 t)} \phi(\xi) d \xi=\int_{E_{2 m}} h_{2 m}(x-\xi, t) \phi(\xi) d \xi
\end{aligned}
$$

with $h_{2 m}$ given by

$$
h_{2 m}(x-\xi, t)=(4 \pi t)^{-m} e^{-a^{2} t} e^{-\|x-\xi\|_{2 m}^{2} / 4 t}
$$

From (2.2), it follows that the kernel function of problem (3.1) is defined by

$$
\begin{aligned}
k_{2 m}(x, y) & =\frac{y}{\sqrt{\pi}} \int_{0}^{\infty} e^{-y^{2} \sigma} \sigma^{-1 / 2} \pi^{-m} \sigma^{m} e^{-\|x\|_{2 m}^{2} \sigma} e^{-a^{2} / 4 \sigma} d \sigma \\
& =\frac{y}{\pi^{m+1 / 2}} \int_{0}^{\infty} \sigma^{m-1 / 2} e^{-r \sigma} e^{-a^{2} /(4 \sigma)} d \sigma \\
& =\frac{y}{\sqrt{\pi}}\left(-\pi^{-1} D_{r}\right)^{m} \int_{0}^{\infty} \sigma^{-1 / 2} e^{-r \sigma} e^{-a^{2} /(4 \sigma)} d \sigma
\end{aligned}
$$

Using the Laplace transform formula number 6 on page 22 of [13], we obtain the following theorem. 
THEOREM 3.1. The kernel $k_{2 m}(x, y)$ for the Dirichlet problem (3.1) can be expressed by the ascent type derivative formula

$$
k_{2 m}(x, y)=y\left(-\pi^{-1} D_{r}\right)^{m} r^{-1 / 2} e^{-a r^{1 / 2}}
$$

with $r$ replaced by $y^{2}+\|x\|_{2 m}^{2}$ after carrying out the $m$ differentiations.

Alternatively, let $F_{m}(r)=\left(-\pi^{-1} D_{r}\right)^{m} r^{-1 / 2} e^{-a r^{1 / 2}}$. Then $k_{2 m}(x, y)=y F_{m}\left(r_{2 m}\right)$ where $r_{2 m}=y^{2}+\|x\|_{2 m}^{2}$. The ascent from the kernel $k_{2 m-2}(x, y)$ to the kernel $k_{2 m}(x, y)$ can be implemented as follows. Start with $k_{2 m-2}(x, y)=y F_{m-1}\left(r_{2 m-2}\right)$. Now replace $r_{2 m-2}$ in the last member of this by $r$ and then operate on this newlyobtained term by the operator $\left(-\pi^{-1} D_{r}\right)$. After completing this, replace $r$ by $r_{2 m}$ and this yields $k_{2 m}(x, y)$. Similar discussions can be given after the theorems to follow.

Next, suppose that at least one of the $c_{j}$ in the problem (1.1), with $n=2 m$, is not zero. Associated with this problem is the generalized heat problem

$$
\begin{aligned}
& u_{t}(x, t)=\Delta_{2 m} u(x, t)+\sum_{j=1}^{2 m} 2 c_{j} \dot{D}_{j} u(x, y)-a^{2} u(x, y)=0, \quad y>0 \\
& u(x, 0+)=\phi(x) .
\end{aligned}
$$

A solution of this is given by the integral formula

$$
u(x, t)=\int_{E_{2 m}} H_{2 m}(x-\xi, t) \phi(\xi) d \xi,
$$

in which, by (2.6) and some simplification,

$$
\begin{aligned}
H_{2 m}(x-\xi, t) & =(4 \pi t)^{-m} e^{-a^{2} t}\left\{e^{\sum_{j=1}^{2 m} 2 c_{j} t D_{j}} e^{-\|x-\xi\|_{2 m}^{2} /(4 t)}\right\} \\
& =(4 \pi t)^{-m} e^{-a^{2} t} e^{-\sum_{j=1}^{2 n}\left(x_{j}-\xi_{j}+2 c_{j} t\right)^{2} /(4 t)} \\
& =(4 \pi t)^{-m} e^{-\sum_{j=1}^{2 m} c_{j}\left(x_{j}-\xi_{j}\right)} \cdot e^{-\left(a^{2}+\sum_{j=1}^{2 m} c_{j}^{2}\right) t} e^{-\|x-\xi\|_{2 m}^{2} /(4 t)} .
\end{aligned}
$$

We obtain the kernel function $\tilde{k}_{2 m}(x, y)$ for the Dirichlet (3.6) by applying the transmutation formula (2.2) to $H_{2 m}(x, t)$. If we introduce this "heat" kernel into (2.2) and carry out the necessary algebraic simplifications, we find

$$
\tilde{k}_{2 m}(x, y)=e^{-\sum_{j=1}^{2 m} c_{j} x_{j}} \frac{y}{\sqrt{\pi}} \pi^{-m} \int_{0}^{\infty} \sigma^{m-1 / 2} e^{-r \sigma} e^{-b / \sigma} d \sigma,
$$

where $r$ has the same meaning as before and where $b=\left(a^{2}+\sum_{j=1}^{2 m} c_{j}^{2}\right) / 4$. The integral in the last member of this has the same form as the integral in the last member of (3.4). With a similar type of evaluation, we have the following theorem. 
THEOREM 3.2. The kernel $\tilde{k}_{2 m}(x, y)$ associated with the Dirichlet problem (1.1) with $n=2 m$ is given by the derivative ascent-type formula

$$
\tilde{k}_{2 m}(x, y)=e^{-\sum_{j=1}^{2 m} c_{j} x_{j}} y\left(-\pi^{-1} D_{r}\right)^{m} r^{-1 / 2} e^{-\lambda r^{1 / 2}},
$$

in which $r$ is replaced by $y^{2}+\|x\|_{2 m}^{2}$ and $\lambda$ is replaced by $\left(a^{2}+\sum_{j=1}^{2 m} c_{j}^{2}\right)^{1 / 2}$ after carrying out the $m$ differentiations.

Note that this differs from the right member of (3.5) only by the factor $e^{-\sum_{j=1}^{2 m} c_{j} x_{j}}$ and the replacement of $a$ in (3.5) by $\lambda$. We call on this observation in Sections 4 and 5 .

\section{Kernel for (1.1) when $n=2 m+1$}

In analogy with the previous section, we first consider the Dirichlet problem

$$
\begin{aligned}
& v_{y y}(x, y)+\Delta_{2 m+1} v(x, y)-a^{2} v(x, y)=0, \quad y>0 \\
& v(x, 0+)=\phi(x) .
\end{aligned}
$$

A heat problem associated with this is given by

$$
\begin{aligned}
& u_{t}(x, t)=\Delta_{2 m+1} u(x, t)-a^{2} u(x, t)=0, \quad t>0 \\
& u(x, 0+)=\phi(x) .
\end{aligned}
$$

Just as in Section 3, the solution function $u(x, t)$ of this can be expressed as

$$
u(x, t)=\int_{E_{2 m+1}} h_{2 m+1}(x-\xi, t, a) \phi(\xi) d \xi,
$$

where the $h_{2 m+1}$ function is as in (3.3) but with $2 m$ replaced with $2 m+1$. Applying the transmutation (2.2) to this "heat" kernel, we obtain the Dirichlet kernel $k_{2 m+1}$ for (4.1):

$$
\begin{aligned}
k_{2 m+1}(x, y) & =\frac{y}{\sqrt{\pi}} \int_{0}^{\infty} e^{-y^{2} \sigma} \sigma^{-1 / 2} e^{-a^{2} /(4 \sigma)}(\sigma / \pi)^{m+1 / 2} e^{-\sigma\|x\|_{2 m+1}^{2}} d \xi \\
& =\frac{y}{\pi}\left(-\pi^{-1} D_{r}\right)^{m} \int_{0}^{\infty} e^{-r \sigma} e^{-a^{2} /(4 \sigma)} d \sigma \\
& =\frac{y}{\pi}\left(-\pi^{-1} D_{r}\right)^{m}\left\{a r^{-1 / 2} K_{1}\left(a r^{1 / 2}\right)\right\},
\end{aligned}
$$

where $K_{1}$ denotes a modified Bessel function (see [11]). The last member of (4.4) follows from the third member by applying formula number 8 , page 22 of [13].

THEOREM 4.1. The kernel $k_{2 m+1}(x, y)$ associated with the Dirichlet problem (4.1) is given by the last member of (4.4) with $r$ replaced by $y^{2}+\|x\|_{2 m+1}^{2}$ after carrying out the $m$ differentiations. 
In analogy with the deduction of Theorem 3.2, we can similarly prove the following theorem.

THEOREM 4.2. The kernel $\tilde{k}_{2 m+1}(x, y)$ associated with the Dirichlet problem (1.1) with $n=2 m+1$ is given by

$$
\tilde{k}_{2 m+1}(x, y)=\frac{y}{\pi} e^{-\sum_{j=1}^{2 m+1} c_{j} x_{j}}\left(-\pi^{-1} D_{r}\right)^{m}\left\{\lambda r^{-1 / 2} K_{1}\left(\lambda r^{1 / 2}\right)\right\}
$$

in which $r$ is replaced by $y^{2}+\|x\|_{2 m+1}^{2}$ and $\lambda$ is replaced by $\left(a^{2}+\sum_{j=1}^{2 m+1} c_{j}^{2}\right)^{1 / 2}$ after carrying out the $m$ differentiations.

Finally, let us note a connection between the ascent formulas (3.5) and (4.4) and the descent method. For this purpose, let $X_{n}=\left(x_{1}, x_{2}, \ldots, x_{n}\right), \Sigma_{n}=\left(\sigma_{1}, \sigma_{2}, \ldots, \sigma_{n}\right)$ and $d \Sigma_{n}=d \sigma_{1} d \sigma_{2} \cdots d \sigma_{n}$. Further, let $\phi\left(X_{n}\right)$ denote an arbitrary infinitely differentiable function with compact support in $E_{n}$ (that is, a testing function) and let $k_{n}\left(X_{n}, t\right)$ denote the kernel in (3.5) or (4.5) according as $n$ is even or odd. Then for $n \geq 1$, we have

or

$$
\int_{E_{n+1}} k_{n+1}\left(X_{n+1}-\Sigma_{n+1}, t\right) \phi\left(\Sigma_{n}\right) d \Sigma_{n+1}=\int_{E_{n}} k_{n}\left(X_{n}-\Sigma_{n}, t\right) \phi\left(\Sigma_{n}\right) d \Sigma_{n}
$$

$$
\begin{gathered}
\int_{E_{n}}\left(\int_{-\infty}^{\infty} k_{n+1}\left(X_{n+1}-\Sigma_{n+1}, t\right) d \sigma_{n+1}\right) \phi\left(\Sigma_{n}\right) d \Sigma_{n} \\
=\int_{E_{n}} k_{n}\left(X_{n}-\Sigma_{n}, t\right) \phi\left(\Sigma_{n}\right) d \Sigma_{n} .
\end{gathered}
$$

Since $\phi$ is arbitrary, we obtain the integral formula

$$
\int_{-\infty}^{\infty} k_{n+1}\left(X_{n+1}-\Sigma_{n+1}, t\right) d \sigma_{n+1}=k_{n}\left(X_{n}-\Sigma_{n}, t\right)
$$

which has the "descent" character.

\section{Kernels for GASPT-type Dirichlet problems}

Next, consider the problem

$$
\begin{aligned}
& v_{y y}(x, y)+\frac{\mu}{y} v_{y}(x, y)+\Delta_{n} v(x, y)-a^{2} v(x, y)=0, \quad y>0 ; \\
& v(x, 0+)=\phi(x) .
\end{aligned}
$$

The equation in this is a GASPT-type equation with $\mu<1$. In view of Theorems 3.2 and 4.2 , it is clear that if we obtain kernel functions (5.1) corresponding to the cases 
$n=2 m$ and $n=2 m+1$, then we need only make minor modifications of these to obtain kernels for the Dirichlet problems

$$
\begin{aligned}
& v_{y y}(x, y)+\frac{\mu}{y} v_{y}(x, y)+\Delta_{n} v(x, y)+\sum_{j=1}^{n} c_{j} D_{j} v(x, y)-a^{2} v(x, y)=0, \quad y>0 \\
& v(x, 0+)=\phi(x) .
\end{aligned}
$$

We leave these changes to the reader. We now apply the transmutation (2.3) to the function $h_{2 m}$ in (3.3) and the corresponding function $h_{2 m+1}$ employed in Section 4 along with formula 8 , page 22 of [13].

THEOREM 5.1. The kernel functions $k_{n}^{\mu}(x, y)$ associated with Dirichlet problems involving (5.1) and corresponding respectively to the cases $n=2 m$ and $n=2 m+1$ are given by

$$
\begin{aligned}
& \text { (a) } k_{2 m}^{\mu}(x, y)=\frac{2^{(\mu+1) / 2} y^{1-\mu}}{\Gamma(1-\mu / 2)}\left(-\frac{D_{r}}{\pi}\right)^{m}\left\{a^{(1-\mu) / 2} r^{(\mu-1) / 2} K_{(1-\mu) / 2}\left(a r^{1 / 2}\right)\right\} \\
& \text { (b) } k_{2 m+1}^{\mu}(x, y)=\frac{2^{\mu / 2} y^{1-\mu}}{\sqrt{\pi} \Gamma(1-\mu / 2)}\left(-\frac{D_{r}}{\pi}\right)^{m}\left\{a^{(2-\mu) / 2} r^{(\mu-2) / 2} K_{(2-\mu) / 2}\left(a r^{1 / 2}\right)\right\}
\end{aligned}
$$

in which $r$ is replaced by $y^{2}+\|x\|_{2 m}^{2}$ in (a) after carrying out the $m$ differentiations and by $y^{2}+\|x\|_{2 m+1}^{2}$ in (b) after carrying out the $m$ differentiations.

We note that if $\mu=-2 p$ with $p=0,1, \ldots$ in $(5.2 \mathrm{a})$ or $\mu=1-2 p$ with $p=1,2, \ldots$ in $(5.2 \mathrm{~b})$, then the respective Bessel functions defining the respective kernels have indices that are halves of odd integers. In these cases, the Bessel functions appearing can also be represented as repeated derivatives of a simple quotient involving an exponential function [11].

\section{Application to abstract Dirichlet problems}

Finally, let $X$ be a Banach space and let $A_{j}^{2}, j=1,2, \ldots, 2 m$, denote a set of infinitesimal generators of bounded semigroups $U_{A_{j}}(t)$ in $X$ where the $A_{j}$ are generators of continuous groups $G_{A_{j}}(t)$ in $X$ with $A_{i} A_{j}=A_{j} A_{i}$ for all $i$ and $j$. From (2.1) of [2], it follows that if $\phi_{j} \in D\left(A_{j}^{2}\right)$, the domain of $A_{j}^{2}$ in $X$, then

$$
U_{A_{j}}(t) \phi_{j}=(4 \pi t)^{-1 / 2} \int_{-\infty}^{\infty} e^{-\xi_{j}^{2} / 4 t}\left(G_{A_{j}}\left(\xi_{j}\right) \phi_{j}\right) d \xi_{j}
$$

Now, consider the abstract Dirichlet problem

$$
V_{y y}(y)+\left(\sum_{j=1}^{2 m} A_{j}^{2}\right) V(y)-a^{2} V(y)=0, \quad y>0 ; \quad V(0+)=\phi
$$


$a>0$ and $\phi \in \bigcap_{j=1}^{2 m} D\left(A_{j}^{2}\right)$. We establish that this has a solution in the integral form

$$
V(y)=\int_{E_{2 m}} k_{2 m}(\xi, y, a)\left\{\left(\prod_{j=1}^{2 m} G_{A_{j}}\left(\xi_{j}\right)\right) \phi\right\} d \xi,
$$

where $\xi=\left(\xi_{1}, \xi_{2}, \ldots, \xi_{2 m}\right), d \xi=d \xi_{1} d \xi_{2} \cdots d \xi_{2 m}$ and where the kernel function $k_{2 m}(\xi, y, a)$ is given by $k_{2 m}(\xi, y, a)=y\left(\pi^{-1} D_{r}\right)^{m}\left(r^{-1 / 2} e^{-a r^{1 / 2}}\right)$ with $r$ replaced by $y^{2}+\sum_{j=1}^{2 m} \xi_{j}^{2}$ after completing the differentiations. This kernel function has the same form as the one given by (3.5).

The development of (6.3) follows the approach of Section 3 by starting with the abstract heat problem

$$
\begin{aligned}
u_{t}(t) & =\left(\sum_{j=1}^{2 m} A_{j}^{2}\right) u(t)-a^{2} u(t)=0, \quad t>0 ; \\
u(0+) & =\phi .
\end{aligned}
$$

This has the semigroup solution form $u(t)=e^{-a^{2} t}\left(\prod_{j=1}^{2 m} U_{A_{j}}(t)\right) \phi$. By calling upon the connecting formula (6.1) for each of the $U_{A_{j}}(t)$, we find, after changing orders of integration, that this formula for $u(t)$ becomes

$$
u(t)=(4 \pi t)^{-m} \int_{E_{2 m}} e^{-\left(\sum_{j=1}^{2 m} \xi_{j}^{2}\right) / 4 t}\left[\left(\prod_{j=1}^{2 m} G_{A_{j}}\left(\xi_{j}\right)\right) \phi\right] d \xi .
$$

Then (6.3) follows by applying the transmutation formula (2.2) to this. We have

$$
\begin{aligned}
V(y) & =\pi^{-1 / 2} y \int_{0}^{\infty} \sigma^{-1 / 2} e^{-\sigma y^{2}} u(1 / 4 \sigma) d \sigma \\
& =\frac{y}{\pi^{m+1 / 2}} \int_{E_{2 m}}\left(\int_{0}^{\infty} \sigma^{m-1 / 2} e^{-\sigma\left(y^{2}+\sum_{j=1}^{2 m} \xi_{j}^{2}\right)} e^{-a^{2} / 4 \sigma} d \sigma\right)\left[\left(\prod_{j=1}^{2 m} G_{A_{j}}\left(\xi_{j}\right)\right) \phi\right] d \xi .
\end{aligned}
$$

Following the procedure of Section 3 and using the Laplace transforms employed there, the reader can readily show that the last member of this reduces to (6.3). For a specific example of this, consider the Dirichlet problem

$$
\begin{aligned}
& V_{y y}(x, y)+\left(x_{1} \frac{\partial}{\partial x_{1}}\right)^{2} V(x, y)+\left(x_{2}^{\alpha} \frac{\partial}{\partial x_{2}}\right)^{2} V(x, y)-a^{2} V(x, y)=0, \quad y>0 \\
& V(x, 0)=\phi\left(x_{1}, x_{2}\right),
\end{aligned}
$$

in which $\alpha=2 l /(2 l+1)$ for $l$ a positive integer and where $\phi\left(x_{1}, x_{2}\right)$ is bounded and has continuous derivatives in $x_{1}$ and $x_{2}$. Now the operator $A_{1}=x_{1}\left(\partial / \partial x_{1}\right)$ defines the 
group relation $G_{A_{1}}(t) f\left(x_{1}\right)=f\left(x_{1} e^{t}\right)$ while the operator $A_{2}=x_{2}^{\alpha}\left(\partial / \partial x_{2}\right)$ defines the group relation $G_{A_{2}}(t) g\left(x_{2}\right)=g\left(\left\{x_{2}^{1-\alpha}+(1-\alpha) t\right\}^{1 /(1-\alpha)}\right)$ (see [3, page 104]). Since $2 m=2$ in this problem, it follows by the solution form (6.6) that

$$
\begin{aligned}
k_{2}(\xi, y, a) & =\left.y\left(-\pi^{-1} D_{r}\right)\left(r^{-1 / 2} e^{-a r^{1 / 2}}\right)\right|_{r=y^{2}+\xi_{1}^{2}+\xi_{2}^{2}} \\
& =\frac{y}{2 \pi}\left[\frac{1}{\left(y^{2}+\xi_{1}^{2}+\xi_{2}^{2}\right)^{3 / 2}}+\frac{a}{y^{2}+\xi_{1}^{2}+\xi_{2}^{2}}\right] \cdot e^{-a \sqrt{y^{2}+\xi_{1}^{2}+\xi_{2}^{2}}} .
\end{aligned}
$$

Using this, the group relations noted above and (6.3), we finally obtain

$$
V(x, y)=\int_{E_{2}} k_{2}(\xi, y, a) \phi\left(x_{1} e^{\xi_{1}},\left\{x_{2}^{1-\alpha}+(1-\alpha) \xi_{2}\right\}^{1 /(1-\alpha)}\right) d \xi_{1} d \xi_{2} .
$$

The reader can develop formulas analogous to (6.3) for the problem (6.2) with $2 m$ replaced by $2 m+1$ as well as for abstract versions of Dirichlet problems involving the GASPT equation.

\section{References}

[1] L. R. Bragg, "Hypergeometric operator series and related partial differential equations", Trans. Amer. Math. Soc. 43 (1969) 319-336.

[2] L. R. Bragg, "The ascent method for abstract wave problems", J. Diff. Eqns 38 (1980) 413-421.

[3] L. R. Bragg, "Transmutations that introduce group operators", J. Diff. Eqns 53 (1984) 98-114.

[4] L. R. Bragg, "Transmutations and ascent", Z. Anal. Andwend. 10 (1991) 123-148.

[5] L. R. Bragg and J. W. Dettman, "A class of related Dirichlet and initial value problems", Proc. Amer. Math. Soc. 21 (1969) 50-56.

[6] F. J. Bureau, "Sur l'integration de l'equations des ondes", Bull. Acad. Royale Belgique, Class. Sci. (S. 5) 31 (1945) 610-624 and 651-658.

[7] J. W. Dettman, "Related solution operators in mathematical physics", Appl. Anal. 22 (1986) 243 272.

[8] R. P. Gilbert, "A method of ascent for solving boundary value problems", Bull. Amer. Math. Soc. 75 (1969) 1286-1289.

[9] J. Hadamard, Lectures on Cauchy's problem in linear partial differential equations (Yale Univ. Press, New Haven, Conn., 1923).

[10] V. Kéyantuo, "The Laplace transform and the ascent method for abstract wave equations", 1993, manuscript.

[11] W. Magnes, F. Oberhettinger and R. Soni, Formulas and theorems for the special functions of mathematical physics (Springer-Verlag, New York, 1966).

[12] F. R. Norwood, "Axisymmetric solutions in elastostatics and elastic wave propagation by ascent methods", Trans. Amer. Soc. Mech. Eng., J. Appl. Mech. 21 (1984) 630-635.

[13] G. E. Roberts and H. Kaufman, Table of Laplace transforms (W. B. Saunders, Philadelphia, 1966). 